\title{
Percutaneous endoscopic removal of a residual foreign body at the orbital-cranial region after transorbital penetrating injury: a novel minimally invasive technique
}

\author{
Ying $\mathrm{Yao}^{1 \dagger}$, Fang Shen ${ }^{2 \dagger}$, Aiqin Chen ${ }^{1}$, Guangyu Ying ${ }^{1}$ and Yongjian Zhu ${ }^{{ }^{*}}$
}

\begin{abstract}
Background: The orbit is susceptible to traumatic injuries, and surgical management of transorbital penetrating injury with foreign body retention is challenging due to the associated risks of nervous and vascular injury. Application of multiple imaging modalities is helpful for the preoperative surgical planning, and technical advances in percutaneous endoscopy would render the surgical approach even less invasive.

Case presentation: We present a 60-year-old female patient with a transorbital penetrating injury (TPI) caused by a bamboo stick in her right eye. The bamboo stick was initially pulled out at the emergency room but, 1 month later, massive bleeding from her nose and mouth occurred. CT and CTA revealed a foreign body in the right orbito-cranial region and a traumatic aneurysm at the right internal carotid artery. Endovascular embolization was first delivered to treat the aneurysm, and the remains of the bamboo stick were completely removed with a percutaneous endoscope. The patient was discharged 1 week later.

Conclusions: A careful preoperative examination by multiple imaging modalities, e.g., CT, MRI, and DSA are essential for the correct diagnosis and surgical planning for the patient with a TPI. We minimised the associated risks of vascular and nervous injuries during foreign body removal by sequential application of the endovascular intervention and percutaneous endoscopic technique.
\end{abstract}

Keywords: Wooden foreign body, Percutaneous endoscope, Transorbital penetrating injury

\section{Background}

Although transorbital penetrating injury (TPI) is relatively rare among head injuries, it constitutes a remarkable proportion of penetrating head injury cases, especially in children. Various objects have been reported to penetrate the low resistance orbital corridors and, depending on the velocity of the impacts, they can either penetrate intracranially or confined within the orbit, causing complications of different severities. Physical examination on the affected orbital region is not accessible because of the presence of periorbital soft-tissue swelling and therefore radiological imaging, such as computed tomography (CT) and

\footnotetext{
* Correspondence: neurosurgery@zju.edu.cn

${ }^{\dagger}$ Equal contributors

'Department of Neurosurgery, Second Affiliated Hospital of Zhejiang

University School of Medicine, Hangzhou, China

Full list of author information is available at the end of the article
}

magnetic resonance imaging (MRI), is necessary for the assessment of the extent of injury, identification of intraorbital foreign bodies, accurate diagnoses and guiding appropriate treatment. CT angiography (CTA) and digital subtraction angiography (DSA) could be further performed in cases with evidence of vascular injuries. Despite the lack of a consensus on the optimal clinical pathway for TPI in the literature, TPI usually requires prompt multidisciplinary surgical intervention. In addition to the primary traumas like globe ruptures, vision loss and death due to massive bleeding, intraorbital foreign bodies could also lead to secondary infection if not appropriately managed. The treatment regime needs to be tailored to the anatomical relationship between foreign body and the orbital, ocular, vascular and nervous tissues. We present here a TPI case whose life-threatening vascular 
complication was first treated by the endovascular intervention. Then the residual intraorbital wooden foreign bodies were safely and completely removed by a minimally invasive percutaneous endoscopic procedure.

\section{Case presentation}

A 60-year-old woman presented with right side vision loss after accidentally fell and had a bamboo stick impaling her right orbit. On arrival to a local emergency department, she complained of right eye pain, loss of right eye vision and right side facial numbness. On physical examination, the patient was neurologically intact (Glasgow Coma Scale score was 15). She had a bamboo stick firmly lodged in her right inferior lateral orbit between the globe and zygomatic bone causing right eye proptosis. There was a complete ophthalmoplegia in the right eye with a fixed and dilated right pupil and loss of vision in her right eye. Her left eye was normal. Cranial and orbital CT scanning showed a bamboo stick $71 \mathrm{~mm}$ ln length terminated in the right inferior orbital fissure without significant injury to the globe or fractures in the right inferior orbital rim (Fig. 1a). DSA showed the right carotid artery was partially compressed while leakage of contrast medium was not observed. To prevent fatal bleeding from the carotid artery during foreign body removal, temporary blockage of the right internal carotid flow with balloon technique was performed first before the bamboo stick was successfully removed along its penetration route by pulling its end that was left outside of the orbit (Fig. 1b). Cranial CT performed after pulling out the bamboo stick revealed local hematoma formation, tissue swelling and possible residual foreign bodies (Fig. 1c). The patient refused further hospitalization, and he was discharged and was advised to have a close clinical follow-up.

One month later, she was admitted to our emergency department with complaints of sudden massive bleedings from her mouth and nose for two times. Physical examination revealed signs of hemorrhagic shock because of the acute blood loss $(800-1000 \mathrm{~mL})$. Cranial $\mathrm{CT}$ revealed a foreign body in the right orbito-cranial region, while CTA and DSA showed a 3-mm traumatic aneurysm at the right internal carotid artery. The aneurysm was first treated by emergent endovascular embolization to stop the life-threatening bleeding. Then the patient was taken to the operating room for removal of the residual bamboo stick via transorbital approach with the percutaneous endoscopic procedure (Spinal Endoscope System; Spinendos, Munich, Germany) by members of the neurosurgery department (Additional file 1: Video S1). With the assistance of neuronavigation system, all the remains of the bamboo stick were withdrawn in about $1.5 \mathrm{~h}$, with a blood loss of about $10 \mathrm{~mL}$ (Fig. 1d-f). After total removal of the

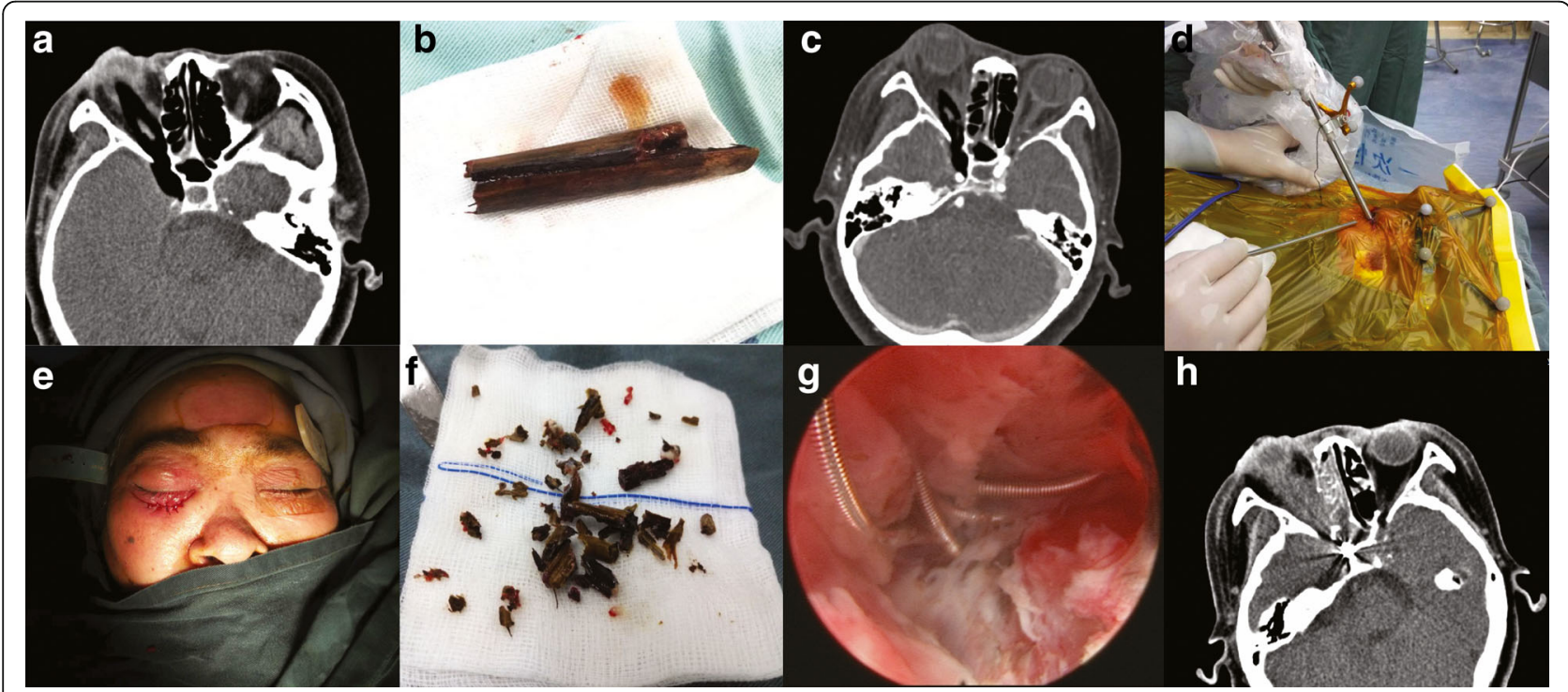

Fig. 1 Percutaneous endoscopic management of orbital-cranial region wooden foreign body in a 60-year-old female patient with transorbital penetrating injury by a bamboo stick. a Cranial CT scanning after injury showed the bamboo terminated in the right inferior orbital fissure without significant injury to the globe. b After temporary blockage of the right internal carotid flow by endovascular balloon, the bamboo stick was retracted along its penetration route. c Cranial CT after bamboo stick removal revealed local hematoma formation, tissue swelling, and possible residual foreign bodies. $\mathbf{d}$ The residual bamboo stick was removed via transorbital approach with the percutaneous endoscopic procedure. e The skin incision and associated injury were minimized by approaching the residual bamboo sticks along its initial penetrating pathway. $\mathbf{f}$ All the remains of the bamboo stick were withdrawn in about $1.5 \mathrm{~h}$ under endoscope. $\mathbf{g}$ The coils used for aneurysm embolization were identified under the endoscopy. $\mathbf{h}$ After endovascular embolization of the traumatic aneurysm and percutaneous endoscopic procedures 1 month after initial injury, cranial CT confirmed complete removal of the residual bamboo sticks 
foreign body, the coils used for aneurysm embolization were also clearly identified under the endoscopy (Fig. 1g). After the operation, symptoms like facial numbness and right eyelid swelling were relieved immediately. Postoperative CT (Fig. 1h) and MRI verified a complete removal of the foreign body. She was discharged 1 week later with a mild contralateral hemiparesis. Written informed consent regarding the publication of this case report and its accompanying images was obtained from the patient.

\section{Discussion and conclusions}

The orbit is fragile and susceptible to traumatic injuries, and TPI accounts for $30-50 \%$ of eye trauma [1]. Surgical managements of TPI have always been challenging due to the closeness of trauma to vital structures like foramina opticum and cavernous sinus [2]. There are several reports on the cerebrovascular complications caused by either initial injury or the surgical intervention to remove the foreign body [3]. CTA and DSA are very helpful for the preoperative surgical planning for foreign body removal and correct diagnosis of vascular complications. If cerebrovascular traumas are indicated, foreign body removal should be arranged either after endovascular treatment or performed in the catheterization procedure rooms. Otherwise, the bleeding during or after removal of the foreign body would be catastrophic.

Wood is the most commonly encountered organic foreign body in TPI. Generally, CT detection of wood may be difficult as its signal would be hypoattenuating in the acute phase, which resembles that of air on CT. But unlike air, wood also has a geometric shape, which might be helpful for differential diagnosis. Besides, the attenuation of wood on CT will change from isoattenuating in the subacute phase to hyperattenuating in the chronic phase. Although bamboo stick is a kind of wood, there is still not much knowledge on the radiological characteristics of a bamboo stick as the intraorbital foreign body [2, 4]. Because of the physical nature of the bamboo (soft and fragile), incomplete removal by direct retraction in the emergency room is highly likely [2]. However, complete removal of organic foreign bodies such as bamboo is important as any remaining pieces could cause an inflammatory response and even severe infection. Also, incomplete removal of the organic foreign body might also lead to massive bleeding due to the erosion of major artery by chronic inflammation process [5]. CT bone windows with a width of -1000 $\mathrm{HU}$ and a soft tissue window level of $-500 \mathrm{HU}$ have been suggested to better identify wooden foreign body and MR would further support the existence of organic foreign bodies by capturing the associated inflammation process [6].
The foreign body causing TPI could penetrate into the brain through either the orbital roof, the superior orbital fissure, or the optic canal [7]. Open surgery via transorbital, transcranial, or combination of both has been used to remove the residual intraocular or intraorbital foreign body [8]. Also, nasal and sinus passages with a nasal endoscopy have also been applied to remove intraorbital foreign body $[6,9,10]$. For our case, instead of using transorbital or transcranial open surgery, a percutaneous endoscopic procedure was chosen to remove the deeply located foreign body. The rationales are as flows: 1 . Surgical injury has been minimised as the endoscope could be inserted through the primary penetrating pathway to approach the foreign body, and all surgical instruments were operated within the endoscopic sheath. 2 . Constant saline irrigation supplied by the endoscopic system could reduce the venous oozing and keep a clear visual field. 3 . A wide variety of grasping forceps is available to ensure the efficient removal of the deeply located foreign bodies while causing minimal tissue damage during the removal. 4. After the successful aneurysm embolization, the risks of its rupture due to endoscopic manipulation are low. 5. The one-month clinical history suggested the wooden foreign body (bamboo stick) might become soft, which makes it easier to be removed piece by piece from the endoscope working canal. To the best of our knowledge, this is the first report on applying the percutaneous endoscopic technique to the removal of an orbital-cranial residual wooden foreign body.

To conclude, the percutaneous endoscopic technique provides better visualization of the foreign body via a minimally invasive approach, and the success of current case suggests this technique could be the treatment of choice for similar patients with intraorbital-cranial retention of the foreign body after TPI.

\section{Additional file}

Additional file 1: Video S1. Percutaneous endoscope - Synchronized Clip. (M4V 65332 kb)

\section{Abbreviations \\ CT: Computed tomography; CTA: CT angiography; DSA: Digital subtraction angiography; MR: Magnetic resonance imaging; TPI: Transorbital penetrating injury \\ Acknowledgements \\ Not applicable \\ Funding \\ This study was supported by Science and Technology Department of Zhejiang Province (Grant No. 2007C33042) and Zhejiang Provincial Natural Science Foundation (Grant No. Y14H090041).}

Availability of data and materials

The authors declare that the data supporting the findings of this study are available within the article and its Additional file. 


\section{Authors' contributions}

YZJ: designed the study and performed the surgery. YY \& FS: drafted the article. AQC \& GYY: collected the data and carried out the follow-up. All authors read and approved the final manuscript

\section{Ethics approval and consent to participate}

Consents to conduct and report of this study was obtained from the Ethics Committee of Second Affiliated Hospital of Zhejiang University School of Medicine.

\section{Consent for publication}

Written informed consent regarding the publication of this case report and its accompanying images was obtained from the patient. Copies of the written consent are available for review upon request.

\section{Competing interests}

The authors declare that they have no competing interests.

\section{Author details}

'Department of Neurosurgery, Second Affiliated Hospital of Zhejiang University School of Medicine, Hangzhou, China. ${ }^{2}$ Department of Orthopaedic Surgery's Spine Division, Affiliated Hospital of Ningbo University School of Medicine, Ningbo, China.

Received: 26 March 2017 Accepted: 20 November 2017

Published online: 04 December 2017

\section{References}

1. Ozkan Arat Y, Arat A, Aydin K. Cerebrovascular complications of transorbital penetrating intracranial injuries. Ulus Travma Acil Cerrahi Derg. 2015;21:271-8.

2. Borkar SA, Garg K, Garg M, Sharma BS. Transorbital penetrating cerebral injury caused by a wooden stick: surgical nuances for removal of a foreign body lodged in cavernous sinus. Childs Nerv. Syst. 2014;30:1441-4.

3. Bodanapally UK, Shanmuganathan K, Boscak AR, Jaffray PM, Van der Byl G, et al. Vascular complications of penetrating brain injury: comparison of helical CT angiography and conventional angiography. J Neurosurg. 2014; 121:1275-83.

4. Matsuyama T, Okuchi K, Nogami K, Hata M, Murao Y. Transorbital penetrating injury by a chopstick-case report. Neurol Med Chir. 2001;41: 345-8.

5. Maruya J, Yamamoto K, Wakai M, Kaneko U. Brain abscess following transorbital penetrating injury due to bamboo fragments-case report Neurol Med Chir. 2002:42:143-6.

6. Jusue-Torres I, Burks SS, Levine CG, Bhatia RG, Casiano R, et al. Wooden Foreign Body in the Skull Base: How Did We Miss It? World Neurosurg. 2016;92:580 e5-9.

7. Balasubramanian C, Kaliaperumal C, Jadun CK, Dias PS. Transorbital intracranial penetrating injury-an anatomical classification. Surg Neurol. 2009:71:238-40.

8. Paiva WS, Monaco B, Prudente M, Soares MS, de Amorim RL, et al. Surgical treatment of a transorbital penetrating brain injury. Clin Ophthalmol. 2010:4 $1103-5$.

9. Feichtinger M, Zemann W, Karcher H. Removal of a pellet from the left orbital cavity by image-guided endoscopic navigation. Int J Oral Maxillofac Surg. 2007;36:358-61.

10. Wu W, Lu SY, Liu CY, Tu Y, Qian Z. Image-guided endoscopic combined with deep lateral Orbitotomy removal of a small foreign body at the deep lateral orbital apex. J Craniofac Surg. 2015;26:e791-3.

\section{Submit your next manuscript to BioMed Central and we will help you at every step:}

- We accept pre-submission inquiries

- Our selector tool helps you to find the most relevant journal

- We provide round the clock customer support

- Convenient online submission

- Thorough peer review

- Inclusion in PubMed and all major indexing services

- Maximum visibility for your research

Submit your manuscript at www.biomedcentral.com/submit 